\title{
A Case of Isolated Adrenocorticotropic Hormone Deficiency Caused by Pembrolizumab
}

\author{
Tomoaki Bekki $^{a}$ Yuji Takakura ${ }^{a}$ Masatoshi Kochia ${ }^{a}$ Yoko Konemori ${ }^{b}$ \\ Kenji Oki $^{b}$ Masayasu Yonedab Hiroyuki Egia Hideki Ohdan ${ }^{a}$ \\ aDepartment of Gastroenterological and Transplant Surgery, Applied Life Sciences, \\ Institute of Biomedical and Health Sciences, Hiroshima University, Hiroshima, Japan; \\ ${ }^{b}$ Department of Endocrinology and Diabetic Medicine, Applied Life Sciences, Institute of \\ Biomedical and Health Sciences, Hiroshima University, Hiroshima, Japan
}

\section{Keywords}

Anti-programmed cell death 1-specific monoclonal antibody - Microsatellite instability-high cancer - Immune-related adverse events · Isolated adrenocorticotropic hormone deficiency

\section{Abstract}

Pembrolizumab (Keytruda ${ }^{\circledR}$ ) is an anti-programmed cell death 1-specific monoclonal antibody that has become the standard second-line chemotherapy for unresectable advanced microsatellite instability-high colorectal cancer. Several immune-related adverse events (irAEs), particularly endocrinopathy, are linked to the administration of pembrolizumab. We report here a case of pembrolizumab-induced isolated adrenocorticotropic hormone deficiency in a patient with metastatic colon cancer. A 65-year-old woman visited our hospital for complaints of fatigue with a recent history of primary resection of cecal mucinous cancer and hepatectomy for liver metastasis 3 years ago. Peritoneal dissemination was detected 2 years after surgery. Several chemotherapeutic regimens of cytotoxic and molecular targeted drugs were administered; however, the metastases progressed gradually. Pembrolizumab monotherapy was started because of resistance to treatment. After 2 cycles of pembrolizumab, the patient was severely fatigued. Laboratory data demonstrated that the cortisol level was extremely low. All the other values were within the normal range. Magnetic resonance imaging indicated no mass in the pituitary gland. From multiple tolerance tests, we diagnosed isolated adrenocorticotropic hormone deficiency caused by pembrolizumab. The patient's symptoms improved promptly with cortisol treatment. An abdominal contrast-enhanced computed tomography scan after 5 cycles of pembrolizumab demonstrated that the size of the perito- 
Bekki et al.: Isolated Adrenocorticotropic Hormone Deficiency Linked to Pembrolizumab

neal dissemination remained unchanged. However, her serum level of carcinoembryonic antigen had decreased to normal levels. Endocrine disorders are very rarely seen as irAEs. Careful laboratory data follow-up is required to inhibit the progression of severe endocrine disorders.

\section{Introduction}

Conventionally, the treatment of unresectable advanced colorectal carcinoma is systemic chemotherapy. Immune checkpoint inhibitors (ICIs) are now recognized as a treatment option. Cytotoxic T-lymphocyte-associated protein 4 (CTLA-4) and programmed cell death 1 (PD-1) are the first well-recognized checkpoints that negatively regulate $\mathrm{T}$-cell immune responses [1, 2]. The Food and Drug Administration (FDA) approved pembrolizumab (Keytruda ${ }^{\circledR}$ ), a PD-1 inhibitor, for patients with unresectable or metastatic solid tumors positive for microsatellite instability (MSI)-high or DNA mismatch repair-deficient biomarkers [3].

This is the first example of an FDA approval of a treatment based on a tumor biomarker and not the type of tumor. Pembrolizumab has shown antitumor activity in a variety of tumors such as non-small cell lung carcinoma, renal carcinoma, urothelial carcinoma, Hodgkin's lymphoma, head and neck carcinoma, and mismatch repair-deficient colorectal carcinoma [4-8]. ICIs have shown autoimmune and inflammatory effects due to increased T-cell activation, which are defined as immune-related adverse events (irAEs) [9-11]. Pembrolizumab has become a mainstay in the treatment for unresectable and advanced MSI-high colorectal carcinoma. Therefore, the frequency with which irAEs will be experienced is also likely to increase. Various organ measurements reveal that organs such as the gastrointestinal tract, endocrine glands, skin, and liver are influenced by ICIs [12].

The incidence of endocrine disorders is low for anti-PD-1 monotherapy [13]. In addition, cases of isolated adrenocorticotropic hormone deficiency are very rare. We present a case of isolated adrenocorticotropic hormone deficiency caused by pembrolizumab in a patient with peritoneal dissemination of mucinous adenocarcinoma of the cecum.

\section{Case Report}

A 65-year-old Japanese woman had previously undergone multiple treatments for metastatic cecal cancer. Her original lesion was discovered 3 years earlier when she had laparoscopic ileocecal resection with D3 lymph node dissection for the primary site. After surgery, adjuvant chemotherapy with CAPOX (capecitabine/oxaliplatin) was administered because of the final stage III diagnosis. Abdominal contrast-enhanced computed tomography (CT) after 3 months detected a solitary liver metastasis despite the adjuvant chemotherapy; hence, she had laparoscopic partial hepatectomy for liver metastasis. Then, 1.5 years after the hepatectomy, peritoneal metastases were detected by abdominal contrast-enhanced CT (Fig. 1).

Therefore, several lines of chemotherapy with anti-vascular endothelial growth factor antibody were administered for 1 year. However, as her disease progressed despite these treatments (Fig. 2), she was started on pembrolizumab at $200 \mathrm{mg}$ because the primary resected specimen revealed that the tumor was MSI-high. After 2 cycles of pembrolizumab, the patient experienced severe fatigue. She had no other complaints such as nausea, anorexia, or abdominal pain. Laboratory data showed a decrease in cortisol $(0.5 \mu \mathrm{g} / \mathrm{dL})$ and adrenocorticotrophic hormone $(\mathrm{ACTH})$ levels $(3.0 \mathrm{pg} / \mathrm{mL})$. Although the patient did not show any signs 
Bekki et al.: Isolated Adrenocorticotropic Hormone Deficiency Linked to Pembrolizumab
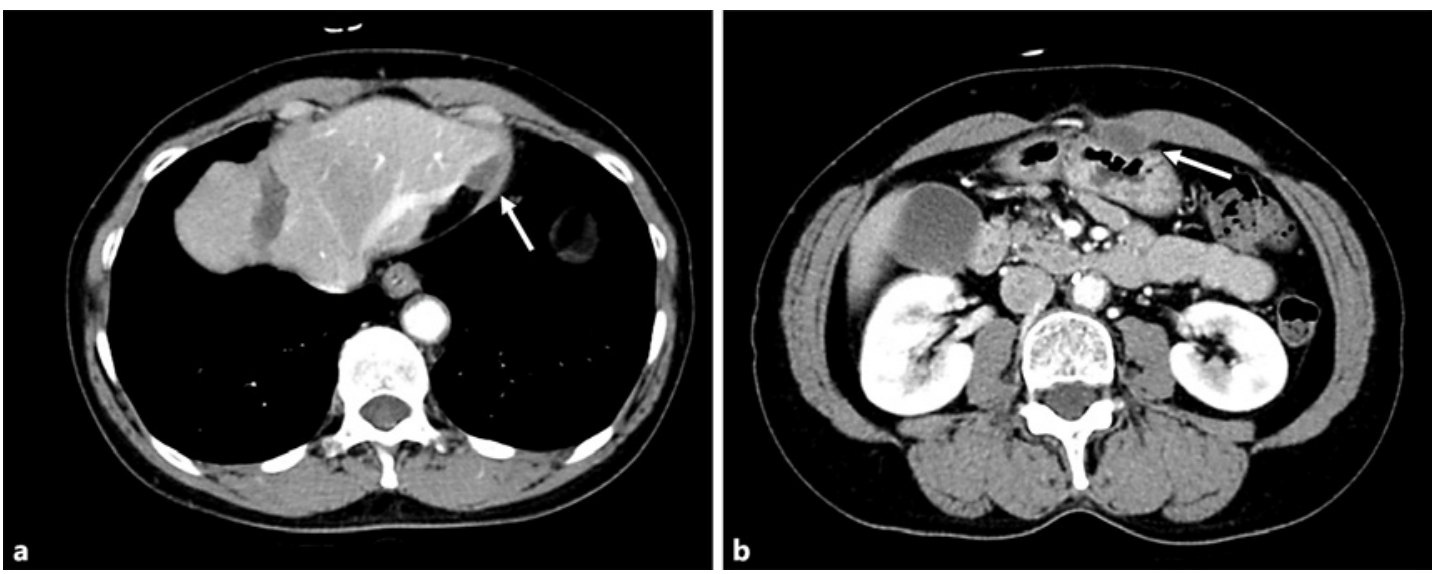

Fig. 1. a, b Abdominal contrast-enhanced CT findings before chemotherapy with cytotoxic and molecular targeted drugs. Low-density areas (white arrows) suspected of being peritoneal dissemination were detected.
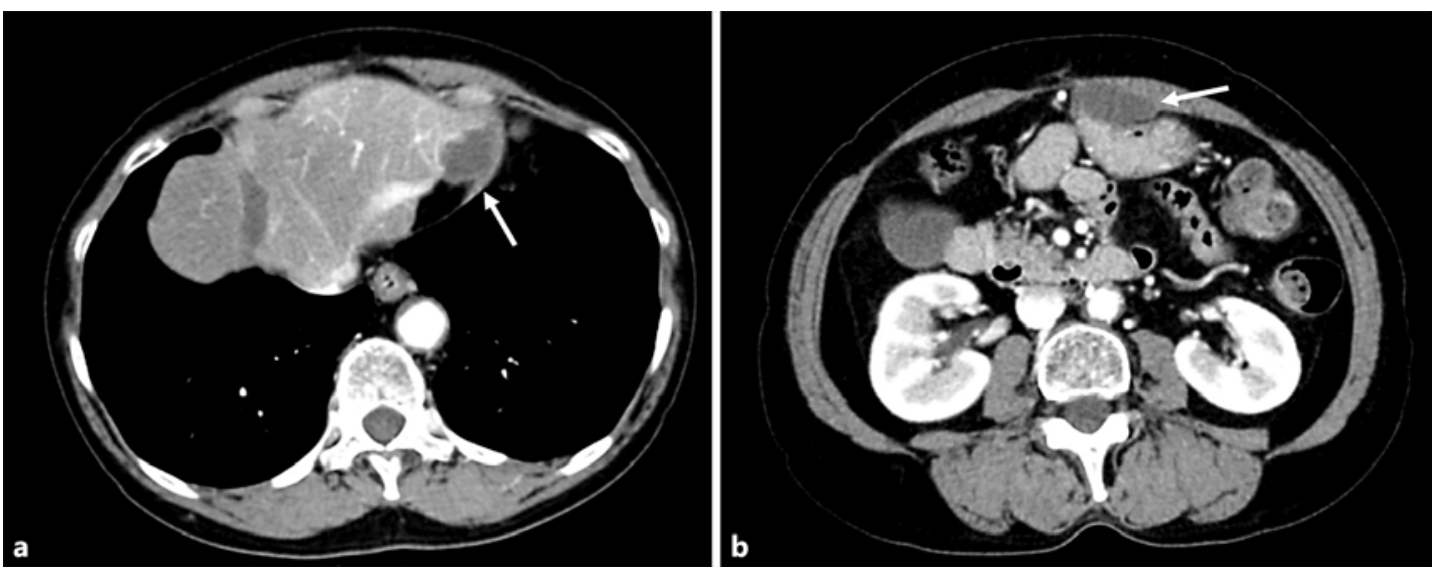

Fig. 2. a, b Abdominal contrast-enhanced CT findings after 5 cycles of chemotherapy with cytotoxic and molecular targeted drugs. The size of peritoneal dissemination (white arrows) had increased.

of anemia and neutropenia, additional examinations were performed during hospitalization due to suspected irAEs. She was suspected with having secondary adrenal insufficiency because of basal blood test findings including low ACTH and cortisol levels.

The magnetic resonance imaging (MRI) findings revealed no remarkable findings in the pituitary gland or hypothalamus (Fig. 3). An anterior pituitary function test by combined intravenous administration of the four hypothalamic releasing hormones showed that the levels were within the normal range, except for the corticotropin-releasing hormone load. ACTH and cortisol showed no response to corticotropin-releasing hormone loading, while the insulin tolerance test demonstrated not only ACTH hyposecretion but also a normal reaction of the growth hormone. The patient was diagnosed with an isolated adrenocorticotropic hormone deficiency. An irAE induced by pembrolizumab was the most likely cause of this condition. The patient started hydrocortisone therapy, which immediately relieved her fatigue. She was discharged on the thirteenth day after admission as her fatigue was reduced. 
Fig. 3. Head MRI findings. There was no tumor in the pituitary gland (white arrow).

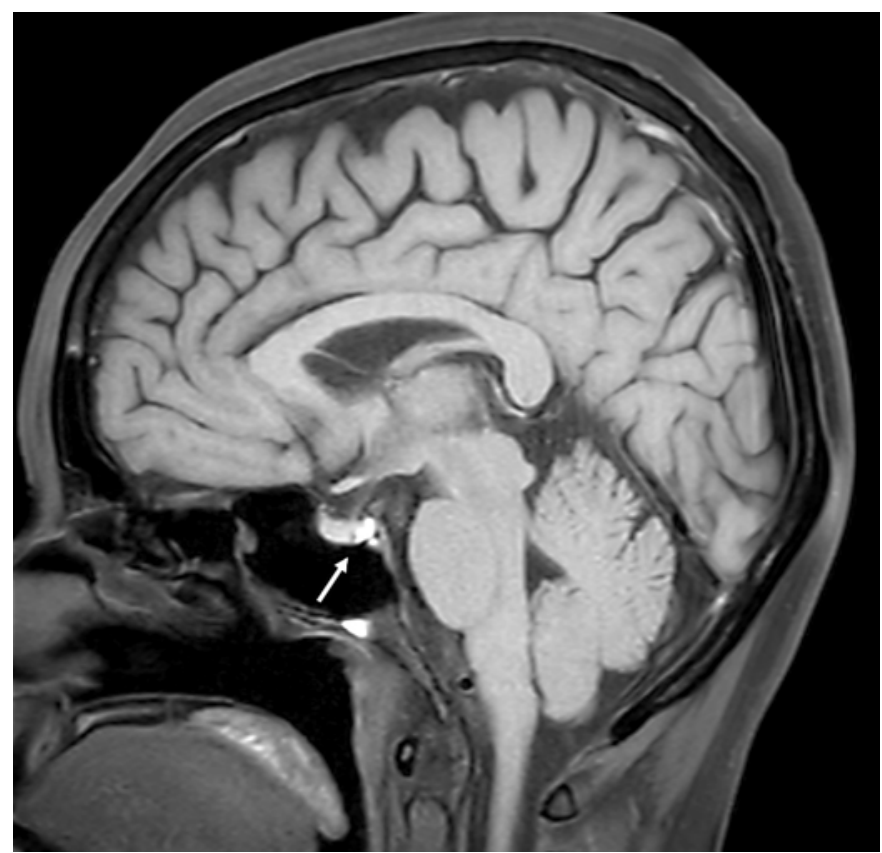

After 5 cycles of pembrolizumab, carcinoembryonic antigen levels were within the normal range, and CT showed that the size of the peritoneal dissemination had progressed slightly (Fig. 4). Pembrolizumab therapy for peritoneal dissemination is still ongoing at the outpatient clinic without relapse of fatigue.

\section{Discussion}

Usually, irAEs develop within a few weeks to months after the first administration of ICIs. Up to $29 \%$ of patients who have been treated with ICIs show signs of an endocrine disorder [14], which tends to occur after the sixth or seventh week, with a median time to onset of 7-20 weeks [15]. Although the likelihood of irAEs is higher in some patients with autoimmune disease, organ or hematopoietic stem cell transplants, chronic viral infection, organ dysfunction, or advanced age, the influences of these conditions remain controversial [16]. However, adrenal insufficiency was found to be very rare. According to past reports, the incidence of adrenal insufficiency was $<4.3 \%[17,18]$. Some case reports have demonstrated adrenal insufficiency caused by nivolumab treatment. However, we could only find 3 previous case reports on adrenal insufficiency during treatment with pembrolizumab. The following search terms were used in PubMed and Google Scholar: "pembrolizumab and adrenal insufficiency." One case was breast cancer treated with pembrolizumab as neoadjuvant chemotherapy [19]. The other cases were stage IV lung cancer treated with pembrolizumab as systemic chemotherapy [20,21]. There are no case reports showing isolated adrenocorticotropic hormone deficiency caused by pembrolizumab.

Adrenal insufficiency presents with nonspecific symptoms such as nausea, vomiting, weakness, fatigue, anorexia, abdominal pain, hypotension, fever, headache, and weight loss. Fatigue, especially, is the most common symptom reported by $16-24 \%$ of patients who were treated with an anti-PD-1-specific monoclonal antibody [13, 22-24]. Careful follow-up is required because of the relationships between dose and irAEs that have been demonstrated 

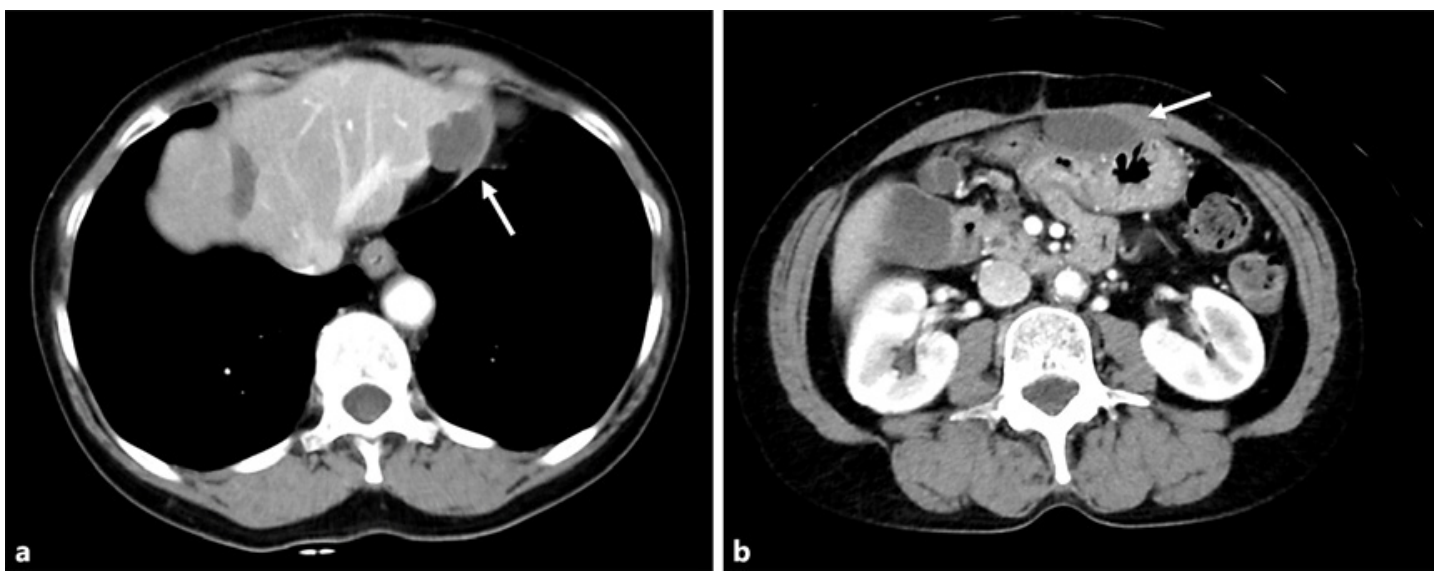

Fig. 4. a, b Abdominal contrast-enhanced CT findings after 5 cycles of chemotherapy with pembrolizumab. The size of peritoneal dissemination (white arrows) was unchanged.

for CTLA-4, whereas toxicities of PD-1 antibodies are reported to be independent of dose [25-27]. In our case study, the onset of fatigue from the first administration was 7 weeks, occurring after only 2 cycles of pembrolizumab. Early diagnosis and starting treatment of irAEs are important to prevent life-threatening complications such as an adrenal crisis $[28,29]$.

If adrenal insufficiency due to ICIs is suspected, it is important to confirm serum cortisol, ACTH, aldosterone, and renin levels. Adrenal insufficiency should be suspected when early morning serum cortisol levels are $<3 \mu \mathrm{g} / \mathrm{dL}$ [30]. Adrenal insufficiency can be classified into two types, primary and secondary. The main differential diagnosis of primary adrenal insufficiency is the appearance of brain metastasis during the treatment of colorectal cancers. MRI with gadolinium contrast is very useful to diagnose the brain metastasis [31]. Other hormone levels - for example, thyroid-stimulating hormone, free thyroxine, free tri-iodothyronine, gonadotrophins, and testosterone - should be checked for an accurate diagnosis [10].

Treatment of adrenal insufficiency consists of systemic corticosteroids. Although most irAEs resolve within weeks to months after starting administration of ICIs [12], they may become severe. Thus, an appropriate treatment should be established. As an example, an initial high dose of corticosteroid of $12 \mathrm{mg} / \mathrm{kg} /$ day for 3 days could be administered, after which the dose would be reduced gradually over a period of at least 1 month [32]. When irAEs do not improve despite the use of adequate steroid medication, treatment can be supplemented with immunosuppressive medicine such as anti-tumor necrosis factor- $\alpha$, mycophenolate mofetil, and antithymocyte immunoglobulins [33]. Endocrine-related AEs are irreversible, and it is important to continue administration of corticosteroids to patients who have developed adrenal insufficiency [34, 35]. Longer-term glucocorticoid therapy may lead to additional complications such as cushingoid features, osteoporosis, glaucoma, debilitating proximal muscle weakness, and opportunistic infections [36] including Aspergillus fumigatus pneumonia, cytomegalovirus hepatitis, and Pneumocystis pneumonia [37-39]. Thus, it is very important for patients being treated with ICIs to be under careful observation.

We could not find any previous reports on isolated adrenocorticotropic hormone deficiency that is caused by pembrolizumab; hence, to the best of our knowledge, this is the first reported case. 
Bekki et al.: Isolated Adrenocorticotropic Hormone Deficiency Linked to Pembrolizumab

\section{Conclusions}

We experienced a rare case of isolated adrenocorticotropic hormone deficiency caused by pembrolizumab. One report showed a positive response from ICI-treated patients who have experienced high-grade irAEs [35]. Additionally, accurate diagnosis and starting treatment early are important to prevent the adrenal insufficiency from becoming severe.

\section{Statement of Ethics}

The patient consented to the reporting of this case in publication.

\section{Disclosure Statement}

We declare no conflicts of interest for this article.

\section{Funding Sources}

This research did not receive any specific grant from funding agencies in the public, commercial, or not-for-profit sectors.

\section{Author Contributions}

T.B., Y.T., K.O., and H.E. wrote the manuscript; Y.T., Y.K., K.O., and M.Y. made the diagnosis and performed treatment. All the authors read and approved the final manuscript.

\section{References}

1 Chambers CA, Kuhns MS, Egen JG, Allison JP. CTLA-4-mediated inhibition in regulation of T cell responses: mechanisms and manipulation in tumor immunotherapy. Annu Rev Immunol. 2001;19:565-94.

2 Ribas A. Tumor immunotherapy directed at PD-1. N Engl J Med. 2012;366:2517-9.

3 Brahmer JR, Tykodi SS, Chow LQ, Hwu WJ, Topalian SL, Hwu P, et al. Safety and activity of anti-PD-L1 antibody in patients with advanced cancer. N Engl J Med. 2012;366:2455-65.

4 Motzer RJ, Rini BI, McDermott DF, Redman BG, Kuzel TM, Harrison MR, et al. Nivolumab for metastatic renal cell carcinoma: results of a randomized phase II trial. J Clin Oncol. 2015;33:1430-7.

5 Powles T, Eder JP, Fine GD, Braiteh FS, Loriot Y, Cruz C, et al. MPDL3280A (anti-PD-L1) treatment leads to clinical activity in metastatic bladder cancer. Nature. 2014;515:558-62.

6 Ansell SM, Lesokhin AM, Borrello I, Halwani A, Scott EC, Gutierrez M, et al. PD-1 blockade with nivolumab in relapsed or refractory Hodgkin's lymphoma. N Engl J Med. 2015;372:311-9.

7 Segal NH, Ou SH, Balmanoukian AS, Fury MG, Massarelli E, Brahmer JR, et al. Safety and efficacy of MEDI4736, an anti-PD-L1 antibody, in patients from a squamous cell carcinoma of the head and neck (SCCHN) expansion cohort. J Clin Oncol. 2015;33(15 Suppl):3011.

8 Le DT, Uram JN, Wang H, Bartlett BR, Kemberling H, Eyring AD, et al. PD-1 blockade in tumors with mismatchrepair deficiency. N Engl J Med. 2015;372:2509-20.

9 Postow MA, Callahan MK, Wolchok JD. Immune checkpoint blockade in cancer therapy. J Clin Oncol. 2015;33: 1974-82.

10 Postow MA. Managing immune checkpoint-blocking antibody side effects. Am Soc Clin Oncol Educ Book. 2015; 76-83.

11 Boutros C, Tarhini A, Routier E, Lambotte O, Ladurie FL, Carbonnel F, et al. Safety profiles of anti-CTLA-4 and anti-PD-1 antibodies alone and in combination. Nat Rev Clin Oncol. 2016;13:473-86.

12 Weber JS, Hodi FS, Wolchok JD, Topalian SL, Schadendorf D, Larkin J, et al. Safety profile of nivolumab monotherapy: a pooled analysis of patients with advanced melanoma. J Clin Oncol. 2017;35:785-92. 
Bekki et al.: Isolated Adrenocorticotropic Hormone Deficiency Linked to

Pembrolizumab

13 Robert C, Schachter J, Long GV, Arance A, Grob JJ, Mortier L, et al. Pembrolizumab versus ipilimumab in advanced melanoma. N Engl J Med. 2015;372:2521-32.

14 González-Rodríguez E, Rodríguez-Abreu D; Spanish Group for Cancer Immuno-Biotherapy (GETICA). Immune checkpoint inhibitors: review and management of endocrine adverse events. Oncologist. 2016;21:804-16.

15 Weber JS, Dummer R, de Pril V, Lebbé C, Hodi FS; MDX010-20 Investigators. Patterns of onset and resolution of immune-related adverse events of special interest with ipilimumab: detailed safety analysis from a phase 3 trial in patients with advanced melanoma. Cancer. 2013;119:1675-82.

16 Johnson DB, Sullivan RJ, Menzies AM. Immune checkpoint inhibitors in challenging populations. Cancer. 2017; 123:1904-11.

17 Doi T, Piha-Paul SA, Jalal SI, Mai-Dang H, Yuan S, Csiki MK, et al. Pembrolizumab (MK-3475) for patients (pts) with advanced esophageal carcinoma: preliminary results from KEYNOTE-028. J Clin Oncol. 2015;33(15 Suppl):4010.

18 Ribas A, Puzanov I, Dummer R, Schadendorf D, Hamid O, Robert C, et al. Pembrolizumab versus investigatorchoice chemotherapy for ipilimumab-refractory melanoma (KEYNOTE-002): a randomised, controlled, phase 2 trial. Lancet Oncol. 2015;16:908-18.

19 Oristrell G, Bañeras J, Ros J, Muñoz E. Cardiac tamponade and adrenal insufficiency due to pembrolizumab: a case report. Eur Heart J Case Rep. 2018;2:yty038.

20 Hanna RM, Selamet U, Bui P, Sun SF, Shenouda O, Nobakht N, et al. Acute kidney injury after pembrolizumabinduced adrenalitis and adrenal insufficiency. Case Rep Nephrol Dial. 2018;8:171-7.

21 Boudjemaa A, Rousseau-Bussac G, Monnet I. Late-onset adrenal insufficiency more than 1 year after stopping pembrolizumab. J Thorac Oncol. 2018;13:e39-e40.

22 Garon EB, Rizvi NA, Hui R, Leighl N, Balmanoukian AS, Eder JP, et al. Pembrolizumab for the treatment of nonsmall-cell lung cancer. N Engl J Med. 2015;372:2018-28.

23 Borghaei H, Paz-Ares L, Horn L, Spigel DR, Steins M, Ready NE, et al. Nivolumab versus docetaxel in advanced nonsquamous non-small-cell lung cancer. N Engl J Med. 2015;373:1627-39.

24 Robert C, Long GV, Brady B, Dutriaux C, Maio M, Mortier L, et al. Nivolumab in previously untreated melanoma without BRAF mutation. N Engl J Med. 2015;372:320-30.

25 Maker AV, Yang JC, Sherry RM, Topalian SL, Kammula US, Royal RE, et al. Intrapatient dose escalation of antiCTLA-4 antibody in patients with metastatic melanoma. J Immunother. 2006;29:455-63.

26 Brahmer JR, Drake CG, Wollner I, Powderly JD, Picus J, Sharfman WH, et al. Phase I study of single-agent antiprogrammed death-1 (MDX-1106) in refractory solid tumors: safety, clinical activity, pharmacodynamics, and immunologic correlates. J Clin Oncol. 2010;28:3167-75.

27 Topalian SL, Hodi FS, Brahmer JR, Gettinger SN, Smith DC, McDermott DF, et al. Safety, activity, and immune correlates of anti-PD-1 antibody in cancer. N Engl J Med. 2012;366:2443-54.

28 Weber JS, Yang JC, Atkins MB, Disis ML. Toxicities of immunotherapy for the practitioner. J Clin Oncol. 2015; 33:2092-9.

29 Brahmer JR, Lacchetti C, Schneider BJ, Atkins MB, Brassil KJ, Caterino JM, et al.; National Comprehensive Cancer Network. Management of immune-related adverse events in patients treated with immune checkpoint inhibitor therapy: American Society of Clinical Oncology clinical practice guideline. J Clin Oncol. 2018;36: 1714-68.

30 Hägg E, Asplund K, Lithner F. Value of basal plasma cortisol assays in the assessment of pituitary-adrenal insufficiency. Clin Endocrinol (Oxf). 1987;26:221-6.

31 Moses AM, Clayton B, Hochhauser L. Use of T1-weighted MR imaging to differentiate between primary polydipsia and central diabetes insipidus. AJNR Am J Neuroradiol. 1992;13:1273-7.

32 Champiat S, Lambotte O, Barreau E, Belkhir R, Berdelou A, Carbonnel F, et al. Management of immune checkpoint blockade dysimmune toxicities: a collaborative position paper. Ann Oncol. 2015;27:559-74.

33 Naidoo J, Page D, Li B, Connell LC, Schindler K, Lacouture ME, et al. Toxicities of the anti-PD-1 and anti-PD-L1 immune checkpoint antibodies. Ann Oncol. 2015;26:2375-91.

34 Lemiale V, Meert AP, Vincent F, Darmon M, Bauer PR, Van de Louw A, et al.; Groupe de Recherche en Reanimation Respiratoire du patient d'Onco-Hématologie (Grrr-OH). Severe toxicity from checkpoint protein inhibitors: what intensive care physicians need to know? Ann Intensive Care. 2019;9:25.

35 Weber JS, Postow M, Lao CD, Schadendorf D. Management of adverse events following treatment with antiprogrammed death-1 agents. Oncologist. 2016;21:1230-40.

36 Huscher D, Thiele K, Gromnica-Ihle E, Hein G, Demary W, Dreher R, et al. Dose-related patterns of glucocorticoid-induced side effects. Ann Rheum Dis. 2009;68:1119-24.

37 Kyi C, Hellmann MD, Wolchok JD, Chapman PB, Postow MA. Opportunistic infections in patients treated with immunotherapy for cancer. J Immunother Cancer. 2014;2:19.

38 Arriola E, Wheater M, Krishnan R, Smart J, Foria V, Ottensmeier C. Immunosuppression for ipilimumab-related toxicity can cause Pneumocystis pneumonia but spare antitumor immune control. Oncoimmunology. 2015;4: e1040218.

39 Uslu U, Agaimy A, Hundorfean G, Harrer T, Schuler G, Heinzerling L. Autoimmune colitis and subsequent CMVinduced hepatitis after treatment with ipilimumab. J Immunother. 2015;38:212-5. 